\title{
Simulation and Design of AlGaAs/InGaAs CCDs based on pHEMT Technology
}

\author{
Hiang T. Tan, Graduate Member, IEEE, Ian C. Hunter, Fellow, IEEE and Christopher M. Snowden, \\ Fellow, IEEE
}

\begin{abstract}
This paper describes the modeling, design and fabrication of quarter-micron double delta doped AlGaAs/InGaAs charge coupled devices whose epitaxial layers and geometry were based around the device structure of commercial pHEMTs. A quasi-two-dimensional physical model has been developed to investigate the properties of this novel 2 dimensional electron gas charge coupled device (2DEG-CCD). This physical model allows the characteristics of the InGaAs transport channel as well as the DC characteristics of the device to be predicted within a reasonable amount of time. This model also shows how 'individual' charge packets can be controllably transferred through the device when appropriate clocking voltages are applied to the gates of the CCD. This capacitive gate structure device is then shown to be successfully fabricated using established GaAs heterostructure fabrication techniques to ensure good repeatability. The DC characteristics of the fabricated charge coupled device delay line are included.
\end{abstract}

Index Terms-2-DEG charge coupled devices, delay line, quasi-2d physical modeling, simulated charge transfer.

\section{INTRODUCTION}

$\mathrm{C}$ HARGE coupled devices are currently widely used in commercial and scientific imaging devices. Silicon based buried channel and surface channel CCDs are best suited for these low frequency applications as the technology is well established but, for microwave frequency applications, alternative device structures such as heterojunction CCDs [1-5] will have to be considered. Heterostructure CCDs are preferred over silicon or gallium arsenide based structures because of the higher electron mobility and higher saturation velocity of carriers in the transport channel of these structures. It has previously been reported that heterostructure CCDs are limited only by the saturation velocity of the carriers which results in an upper frequency limit of approximately $40 \mathrm{GHz}$ [1].

This paper will present a detailed analysis of novel CCD structures that were designed around the epitaxial and geometrical structure of commercial pHEMTs. These structures can then be designed to function as a shift register or a delay line. Realizing a clocked monolithic microwave

Manuscript received ....

Hiang T. Tan is with the Institute of Microwaves and Photonics in the School of Electronic and Electrical Engineering at the University of Leeds, Leeds. LS2 9JT. UK. httan@ieee.org.

Ian C. Hunter is with the Institute of Microwaves and Photonics in the School of Electronic and Electrical Engineering at the University of Leeds, Leeds. LS2 9JT. UK. i.c.hunter@leeds.ac.uk.

Christopher M. Snowden is the Vice-Chancellor and Chief Executive of the University of Surrey, Guildford, Surrey, GU2 7XH,UK, c.snowden@surrey.ac.uk. integrated circuit (MMIC) delay line is of particular interest as it is a key element in the architecture of a tunable MMIC charge transversal filter. These tunable MMIC filters would find many uses in microwave frequency applications such as personal mobile communication devices and in the front end of agile radar communication systems which are always striving to integrate as many components as possible into a small area. This device would allow the comparatively space consuming filtering components to be replaced by integrating them into the existing MMICs found in these devices. These tunable MMIC filters would be cost effective as they could be made using existing GaAs fabrication technology, thus negating the need for separate and expensive fabrication lines.

The quasi-2D physical model developed in this work allows the dependency of the device's behavior on the geometrical and epitaxial buildup of the CCD to be predicted within a few minutes. This then removes the need for time consuming and expensive experimental fabrication processes prior to arriving at a viable prototype design.

\section{DEVICE ARChiteCtURE AND OPERATION}

The simplified structure and the band diagram of two different double delta doped heterostructure CCDs which represent a development and improvement on the buried channel heterojunction CCD reported in [1] are shown in Fig. $1\left(1^{\text {st }}\right.$ structure $)$ and Fig. 2 ( $2^{\text {nd }}$ structure). These two structures have almost identical epitaxial layers with the main difference between them being the location of the Schottky gate contacts. The gate contacts in Fig. 1 are grown on top of an n-type GaAs layer whereas in Fig. 2, the gate contacts are grown on top of an undoped $\mathrm{AlGaAs}$ layer.

From these diagrams, it can be seen that the layer structure and geometrical layout of these devices are extremely similar to that of a double delta doped pseudomorphic HEMT. In the case of the heterostructure CCDs, the length of the gate electrodes are $0.25 \mu \mathrm{m}$ and its chosen gate width is $100 \mu \mathrm{m}$. The length of the interelectrode gap is $1.95 \mu \mathrm{m}$ for the $1^{\text {st }}$ structure and $0.8 \mu \mathrm{m}$ for the $2^{\text {nd }}$ structure. Special care has to be taken when the length of the gate contacts are chosen as this directly affects the time constants of the charge transfer mechanisms [6]. The quarter-micron gates described in this paper are ideal for rapid charge transfer (by large fringing fields and thermal diffusion) making them optimum for high frequency operations.

The InGaAs transport layer is sandwiched between two AlGaAs spacer layers for increased mobility of the carriers. 
The first n-type doped $\left(>10^{18} \mathrm{~cm}^{-3}\right)$ AlGaAs region is located above the first spacer layer and the second doped layer is buried below the second spacer region. The device is completed with ohmic contacts located at the beginning and at the end of the structure. Charge confinement in the transport layer is important in order to ensure that the device has good charge transfer efficiency.

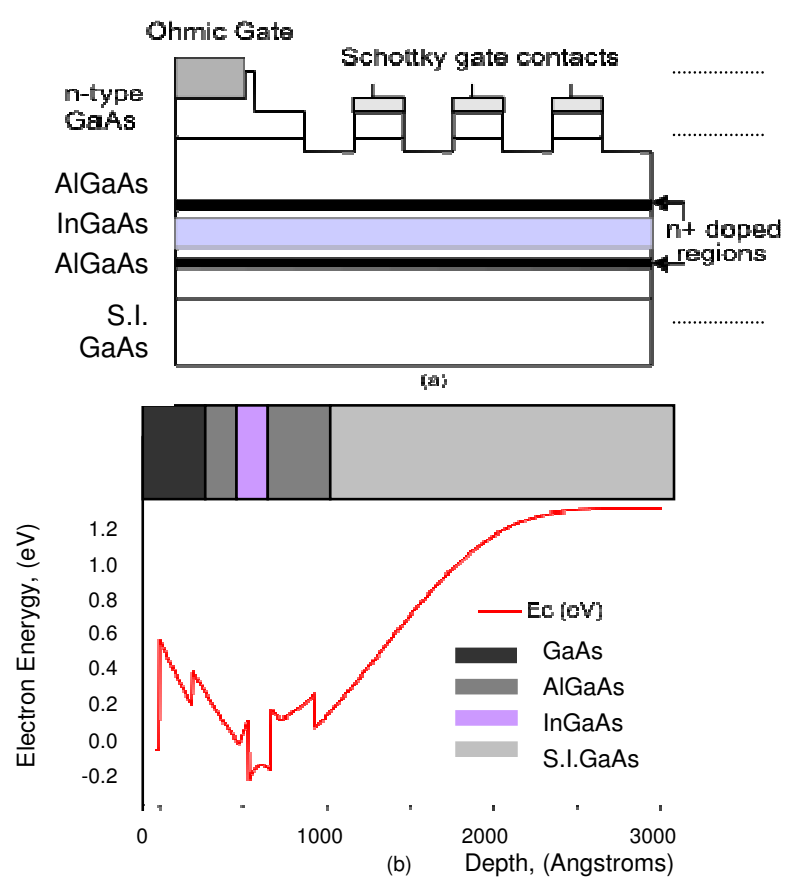

Fig.1 (a) The side schematic of a recessed gate CCD. (b) The simulated conduction band diagram beneath a single Schottky gate contact.
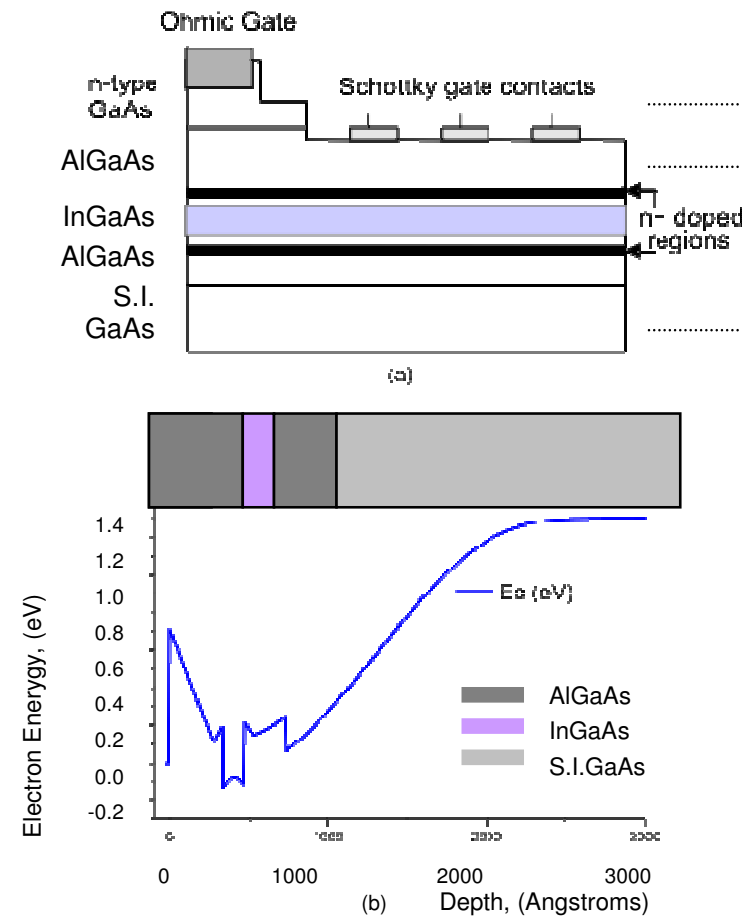

Fig.2 (a) The side schematic of a CCD. (b) The simulated conduction band diagram beneath a single Schottky gate contact.
Conventionally, the analysis of any new charge coupled device structure begins by obtaining an equation that relates the channel potential in the device to the charge packet density in the channel and to the applied gate voltage as it is the 'formation and destruction' of potential wells in the device that provides the mechanism of charge transfer. Under the assumption that channel quantization effects can be neglected, a one-dimensional Poisson's equation is solved in order to obtain a simplified approximation of the two-dimensional electron gas (2DEG)-CCD's channel potential beneath a single Schottky gate. The approximated channel potential equation for the $1^{\text {st }}$ device structure is found to be as follows:

$$
\begin{aligned}
& V_{C H}=\left(\frac{-q Q_{C}}{\varepsilon_{A}}+\frac{q N_{d 2} d_{d 2} \varepsilon_{I}}{\varepsilon_{A}^{2}}\right)\left(\frac{q \varepsilon_{G} d_{G}}{\varepsilon_{A}}+d_{i 1}+d_{d 1}+d_{01}\right)+ \\
& \frac{q N_{d d} d_{G}^{2}}{2 \varepsilon_{G}}-\frac{q N_{d 1} d_{d 1}}{\varepsilon_{A}}\left(\frac{d_{d 1}}{2}+d_{G}+d_{i 1}\right)+\frac{q N_{d 1} d_{d 1}}{\varepsilon_{A}}\left(d_{i 1}+\frac{\varepsilon_{A} d_{G}}{\varepsilon_{A}}\right) \\
& +V_{G}-\phi_{B}+\Delta E_{C}
\end{aligned}
$$

Using a similar approach, the approximated channel potential equation for the $2^{\text {nd }}$ structure is given as follows:

$$
\begin{aligned}
& V_{C H}=\left(\frac{-q Q_{C}}{\varepsilon_{A}}+\frac{q N_{d 2} d_{d 2} \varepsilon_{I}}{\varepsilon^{2}{ }_{A}}\right)\left(d_{i 1}+d_{d 1}+d_{01}\right)+ \\
& \frac{q N_{d 1} d_{d 1}}{\varepsilon_{A}}\left(d_{i 1}+\frac{d_{d 1}}{2}\right)+V_{G}-\phi_{B}+\Delta E_{C}
\end{aligned}
$$

where $d_{\mathrm{G}}$ is the thickness of the doped GaAs layer, $d_{i 1}$ is the thickness of the undoped AlGaAs layer, $d_{\mathrm{d} 1}$ is the thickness of the first delta-doped AlGaAs layer, $d_{01}$ is the thickness of first spacer layer, $d_{\mathrm{C}}$ is the thickness of the transport layer, $d_{02}$ is the thickness of second spacer layer, $d_{\mathrm{d} 2}$ is the thickness of the second delta-doped AlGaAs layer, $\phi_{\mathrm{B}}$ is the Schottky barrier height, $\varepsilon_{\mathrm{G}}$ is the dielectric permittivity for $\mathrm{GaAs}, \varepsilon_{\mathrm{I}}$ is the dielectric permittivity for InGaAs, $\varepsilon_{\mathrm{A}}$ is the dielectric permittivity for $\mathrm{AlGaAs}, \Delta \mathrm{E}_{\mathrm{C}}$ is the conduction band discontinuity, $V_{\mathrm{G}}$ is the gate voltage and $Q_{\mathrm{C}}$ is the electron channel density per unit area. The maximum charge capacity in a potential well for both these device structures can be obtained from these two equations. For the structure in Fig. 1, the potential well's maximum charge capacity is found to be:

$$
\mathrm{Q}_{\mathrm{CMax}}=\frac{\varepsilon_{\mathrm{A}}\left(\mathrm{Vg}_{\mathrm{On} 1}-\mathrm{Vg}_{\mathrm{Off} 1}\right)}{\mathrm{q}\left(\frac{\mathrm{q} \varepsilon_{\mathrm{G}} \mathrm{d}_{\mathrm{G}}}{\varepsilon_{\mathrm{A}}}+\mathrm{d}_{\mathrm{i} 1}+\mathrm{d}_{\mathrm{d} 1}+\mathrm{d}_{01}\right)}
$$

and for the structure in Fig. 2, the potential well's maximum charge capacity is found to be:

$$
\mathrm{Q}_{\mathrm{CMax}}=\frac{\varepsilon_{\mathrm{A}}\left(\mathrm{Vg}_{\mathrm{On} 2}-\mathrm{Vg}_{\mathrm{Off} 2}\right)}{\mathrm{q}\left(\mathrm{d}_{\mathrm{i} 1}+\mathrm{d}_{\mathrm{d} 1}+\mathrm{d}_{01}\right)}
$$


where $\mathrm{Vg}_{\mathrm{On} 1}$ and $\mathrm{Vg}_{\mathrm{On} 2}$ are the maximum achievable positive gate voltages which are normally determined by the Schottky gate contacts (approximately $0.7 \mathrm{~V}$ ) and $\mathrm{Vg}_{\text {Off } 1}$ and $\mathrm{Vg}_{\mathrm{Off} 2}$ are the pinch-off voltages of the $1^{\text {st }}$ and $2^{\text {nd }}$ device structures respectively.

Charge packets in a 2DEG-CCD are normally attracted to wells of higher potential and as these wells fill up, their potential level reduces accordingly, and vice versa, as can be derived from equations 1 and 2 . These equations can be used to plot 'potential well' diagrams as shown in Fig. 3. At time $t_{1}$, the channel potential beneath gates $\mathrm{G} 1$ and $\mathrm{G}+$ become sufficiently positive to attract charge from beneath the input ohmic contact. Then, at time $t_{2}$, the signal charge collected beneath gate $\mathrm{G}+$ gets transferred to the right due to the changing potential beneath these gates. The signal charge beneath gate $\mathrm{G}+$ does not spill back into the potential well beneath the input ohmic contact at time $t_{2}$ because of the barrier formed by gate $\mathrm{G} 1(\mathrm{G}+$ is driven with a clocking signal that has a bigger peak voltage).
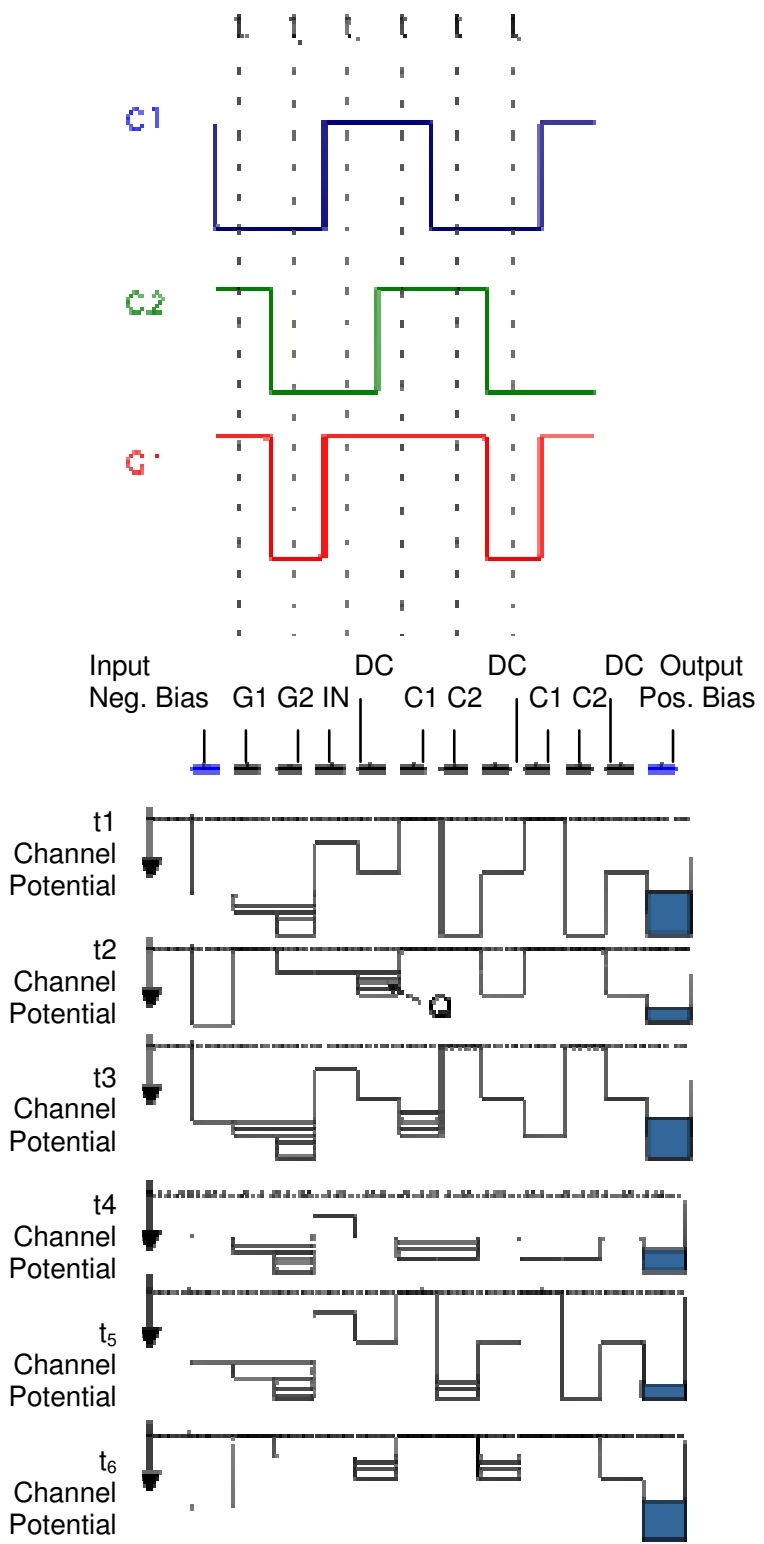

Fig.3. The formation of the potential wells in a simplified 2DEGCCD. These wells are depicted by different snapshots in time $\left(t_{1}-t_{6}\right)$ when a three-phase clocking scheme is applied. G+ is a clock signal which is in phase with clock G1 but with different peak voltages.
The amount of charge that gets transferred forward depends on the amplitude of the input signal. At the next clock cycle, $t_{3}$, the signal charge gets transferred to gate $\mathrm{C} 1$ and then becomes evenly distributed between gates $\mathrm{C} 1$ and $\mathrm{C} 2$ during time $t_{4}$. At time, $t_{5}$, the entire process repeats itself with more charge packets being acquired from beneath the input ohmic gate. The original charge packet in the CCD gets transferred through the device as it is clocked until it is extracted from the output using a source follower setup as described in [7].

\section{QUASI-2D PHYSICAL MODEL}

The physical structures shown in Fig. 1 and Fig. 2 were simulated using a modified version of a quasi-two dimensional pHEMT model (the Leeds Physical Model [810]) to verify the operation of the design. The input data includes information about the process, the delta doping levels in the structure and the cross-sectional geometry. The charge-control model for this simulation self-consistently solves coupled two-dimensional Poisson and time independent Schrödinger equations in order to obtain accurate distributions of the electric field and potential contours in the device's active channel according to the physical parameters (planar or recessed gate structures, number of doped layers) of the device. Once this model has obtained a solution to the coupled Poisson-Schrödinger equations, the results are then stored in look-up tables which are then referenced, when the transport model is solved along the channel of the CCD. The transport model is based on a very fast numerical solution of the continuity, energy and momentum conservation equations which also take account of hot electron transport in these sub-micron gated structures. The transport properties for the materials used in the device were obtained from Monte Carlo simulations and take account of the temperature, doping density, mole fraction and electron energy. Key parameters such as the energy relaxation time as a function of electron energy are extracted from this data. It was found in the original research on HEMT modeling [10] that a quasi-two dimensional approach remains an accurate means of analysing these structures, where the electric field in the undepleted conducting channel is assumed to be onedimensional, whilst retaining a two-dimensional description of the depleted regions of the device. As the model is based upon a fast, accurate and robust solution algorithm, the simulation time is extremely short and can be completed within minutes on a personal computer. A much more detailed description on the workings of this quasi-2D pHEMT model can be found in $[9,11,12]$.

A major change that was made to the basic quasi-2D pHEMT model was the introduction of additional Schottky contacts. For example, an additional gate contact converts this model into a dual-gated one (an initial step towards multi-gated models of the CCD). It should be noted that practical CCDs usually consist of a large number of gate contacts (>30 gates) but for this modeling work, initially only two gates were considered as it greatly shortens the overall simulation time while providing valuable insight into how the chosen epitaxial layers and geometrical layout affects the DC and channel characteristics of the final charge coupled device. 


\section{A. DC Characteristics}

The simulated DC characteristics of the dual gate CCDs are shown in Fig. 4 and Fig. 5. The IV characteristics in Fig. 4 show that the $1^{\text {st }}$ device structure only pinches off when the gate biases exceed approximately -2.7 Volts whereas, the IV curves in Fig. 5 show that the $2^{\text {nd }}$ device structure pinches off at much lower gate voltages (approximately -1.0 Volts). From Fig. 5, it can be seen that each gate independently influences the overall amount of charge that travels through the device.

Due to the larger than normal pinch off voltage of the $1^{\text {st }}$ device structure (Fig. 4), it was decided that the $2^{\text {nd }}$ device structure is more suitable for CCD operations as it is less prone to suffer from off-state breakdown issues. These issues will be addressed in Section V. The charge transfer process in the $2^{\text {nd }}$ device structure is discussed in the following section.

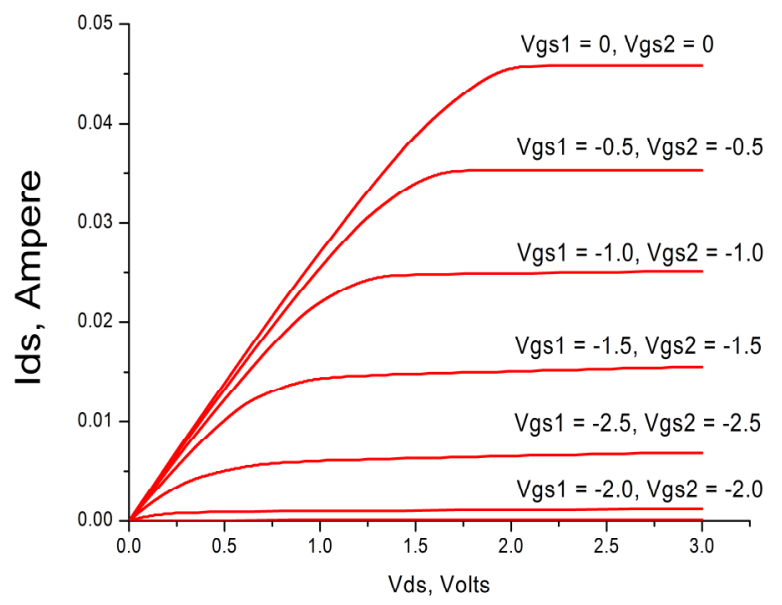

Fig.4. The simulated I-V characteristics for the first device structure shown in Fig. 1.

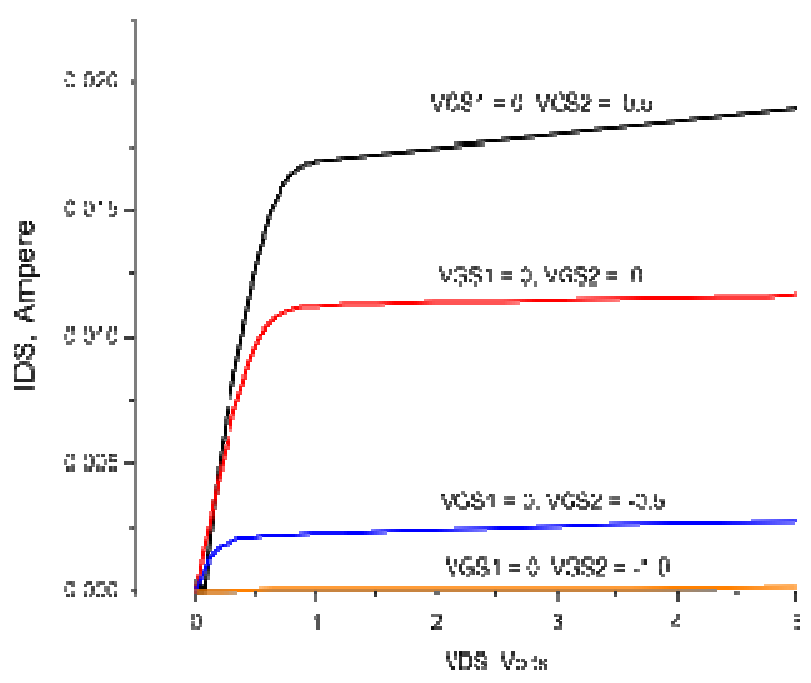

Fig.5. The simulated I-V characteristics for the second device structure shown in Fig. 2 with Vgs1 set to $0 \mathrm{~V}$ and $\mathrm{Vgs} 2$ varied from $0.5 \mathrm{~V}$ till $\mathrm{Vgs} 2=-1.0 \mathrm{~V}$ with a step size of $0.5 \mathrm{~V}$.

\section{B. Charge Transfer}

Most pHEMT models place more emphasis on the DC characteristics of the device but in this modified quasi-2D model, more emphasis is placed on the relationship between the 'potential wells' in the channel and the charge in the channel. The purpose of this simulation is to describe the charge transfer process that occurs in the transport channel thus validating this device structure as a possible CCD delay line. The relationship between the 'potential wells' and the charge in the channel can be seen from Fig. 6 and Fig. 7 under a variety of gate bias conditions.

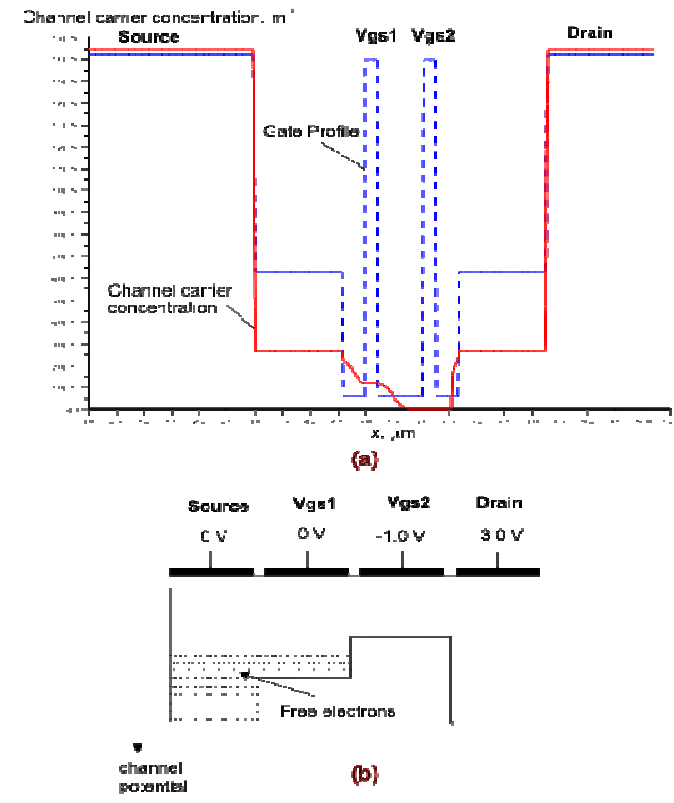

Fig.6. (a) The simulated channel carrier concentration in the $2^{\text {nd }}$ device structure when Vgs1 $=0 \mathrm{~V}$ and Vgs2 $=-1.0 \mathrm{~V}$. (b) The 'potential wells' in the channel under similar biasing conditions.

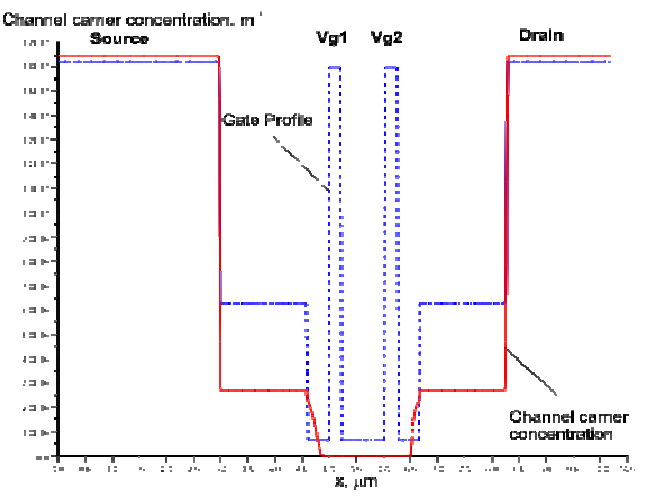

(a)

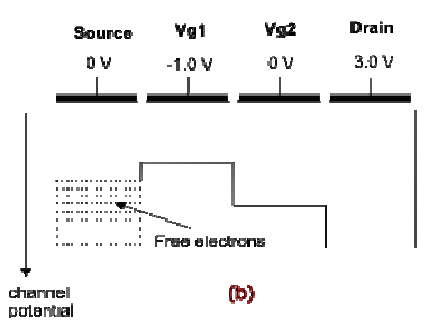

Fig.7. (a) The simulated channel carrier concentration when Vgs1 $=-1.0 \mathrm{~V}$ and $\mathrm{Vgs} 2=0 \mathrm{~V}$. (b) The 'potential wells' in the channel under similar gate biasing conditions. 
In Fig. 6, when a more positive voltage is applied to the first gate, this causes a deeper potential well to form beneath it. In contrast, the second gate has a more negative gate bias thus acting as a barrier to the flow of electrons, effectively constraining the charge packet to remain beneath the first gate contact. In Fig. 7, even though a deeper potential well forms beneath the second gate, no charge packets are attracted to this well due to the barrier created by the shallower potential well beneath the first gate electrode. These diagrams show that by applying the appropriate voltages to the gate contacts, the transfer of the 'individual' charge packets can be accurately controlled. The main significance of these two results is to show that conventional pHEMT device structures potentially could be used to design 2DEG-CCD delay lines or shift registers.

\section{Model Comparison}

The device structures modeled in this paper are novel and thus it is quite difficult to validate the results against conventional existing CCD data. The simulated IV curves for a single gate and a dual gate device based on the first device structure are compared to the experimental IV curves for a 12-gate prototype charge coupled device [4] which has a similar device structure in an attempt to show that a relationship exists between the number of gates and the drain-source currents, IDSS. These IV curves are shown in Fig.8. The impact of larger numbers of gate fingers spaced along a longer channel is to reduce the current level at a given voltage bias point and soften the knee of the I-V characteristic (similar to the transition from single to dual gate FET structures).

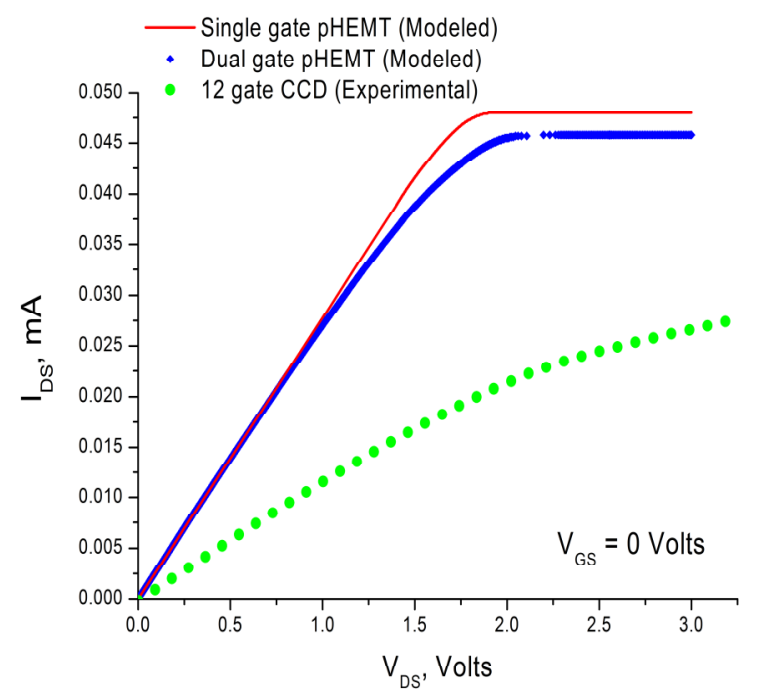

Fig.8. The simulated IV curves for a single gate and dual gate device. Shown also are the measured IV curves obtained from the DC characterization of a 12-gate device.

These results show that as the number of gates in the device increases, the maximum drain-source current decreases accordingly. When a single gate device is modeled, the saturations current, $\mathrm{I}_{\mathrm{DSS}}$ is approximately 48 $\mathrm{mA}$ and when a dual gate structure is modeled, the $\mathrm{I}_{\mathrm{DSS}}$ reduces by $2 \mathrm{~mA}$ to $46 \mathrm{~mA}$. Using this relationship, it was predicted that the $I_{D S S}$ for a 12-gate device would be approximately $24 \mathrm{~mA}$ and from Fig. 8, it can be seen that this prediction is quite close to the reported experimental results.

\section{DEVICE FABRICATION}

Filtronic's $0.25 \mu \mathrm{m}$ pHEMT process was used to fabricate a CCD delay line which was based on the $1^{\text {st }}$ device structure (Fig. 1). The process was modified to allow for multi-gate structures with inter-electrode recesses. These low resistance, Schottky gate structures with silicon nitride, $\mathrm{SiN}$, passivation, were fabricated on 6" GaAs wafers. These gate contacts sit in a dry-etch defined gate recess, while excellent pinch-off uniformity is maintained by a high selectivity etch-stop layer. A multi-layered surface passivation process effectively reduces dispersion in the current-voltage characteristics to a very low level. The size of the actual ' $\mathrm{CCD}$ delay line', shown centrally in Fig. 9, is only $100 \mu \mathrm{m} \times 50 \mu \mathrm{m}$ while the size of the entire test layout is $1.6 \mathrm{~mm} \times 1.6 \mathrm{~mm}$. Bias pads are located at specific points in the MMIC to allow for ease of integration with periphery devices for testing purposes. Biasing circuitry such as decoupling capacitors and inductors are also included. The final fabrication result is extremely accurate as shown in Fig. 10, where the interelectrode gap length of $1.95 \mu \mathrm{m}$ is fabricated with just $\sim 0.01 \mu \mathrm{m}$ error. Fig. 11 shows the ability of the $0.25 \mu \mathrm{m}$ process to produce a uniform multi gate structure over a large area. This is extremely important to ensure a reproducible and predictable CCD operation.

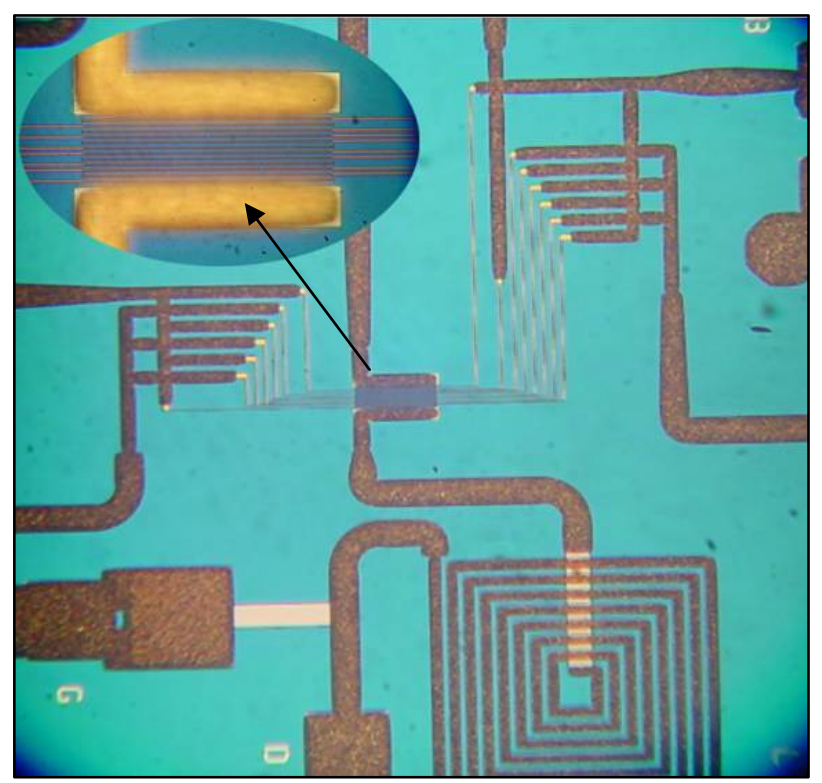

Fig.9. The fabricated test array of the CCD delay line MMIC with emphasis on the actual CCD structure located in the center.

The improved layout for a 13-gate CCD delay line based on the $2^{\text {nd }}$ device structure, which has its charge sensing circuitry (a source follower with a current source and a reset switch) located on-board the same chip, is shown in Fig. 12 whilst the final fabricated device is shown in Fig. 13. 


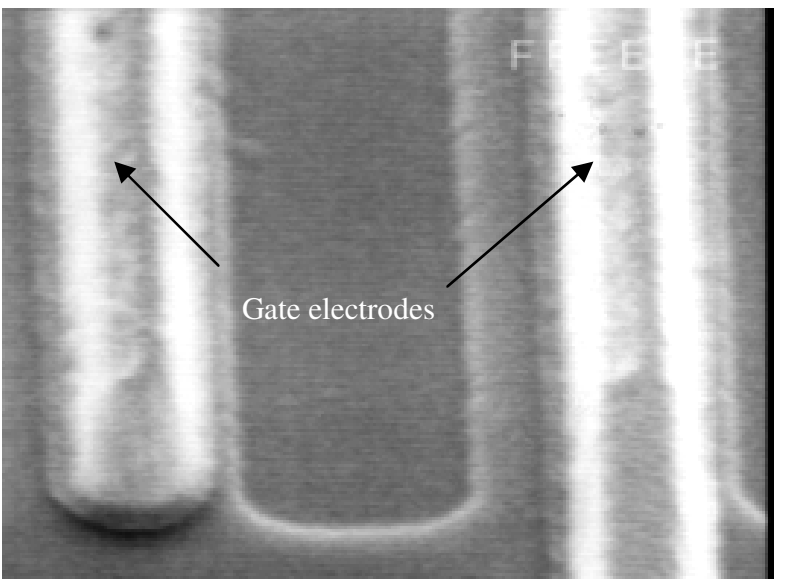

Fig.10. A SEM photomicrograph of two gate electrodes separated by the recessed gap.

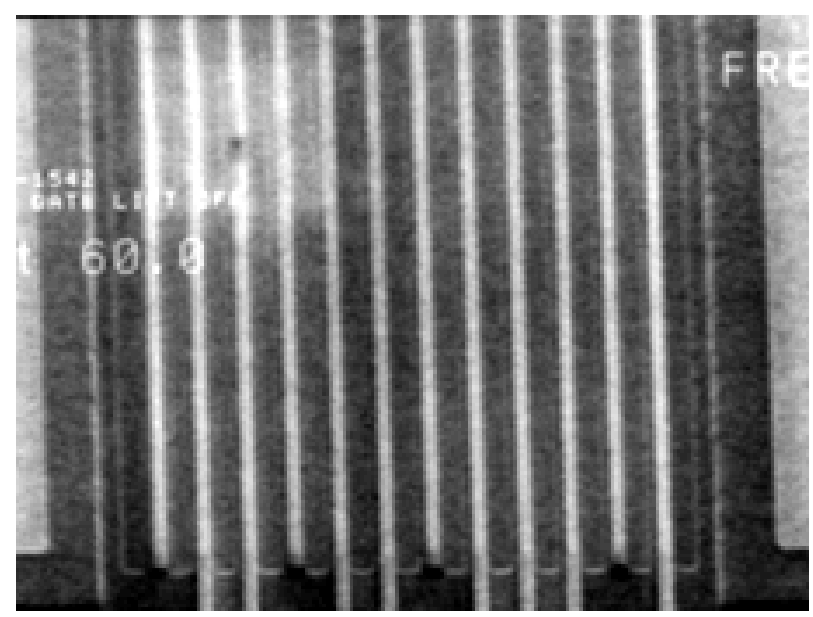

Fig.11. A SEM photomicrograph of the $100 \mu \mathrm{m}$ x $50 \mu \mathrm{m}$ CCD delay line test array. Notice the uniformity of the gates and the interelectrode gaps due to the fabrication process.

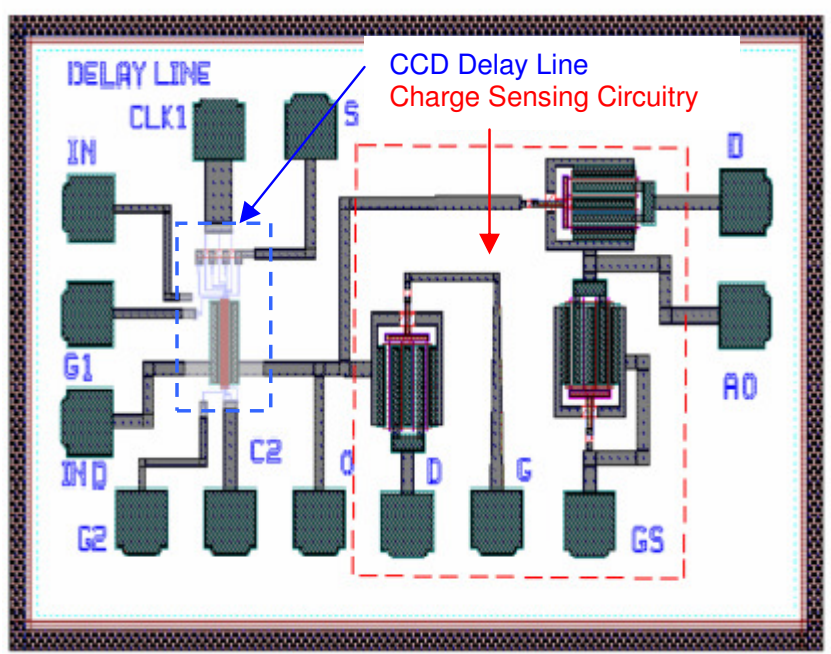

Fig.12. The two dimensional layout of the 13-gate CCD delay line. The charge sensing circuitry which consists of a source follower circuit is 'on-board' the same chip as the CCD.

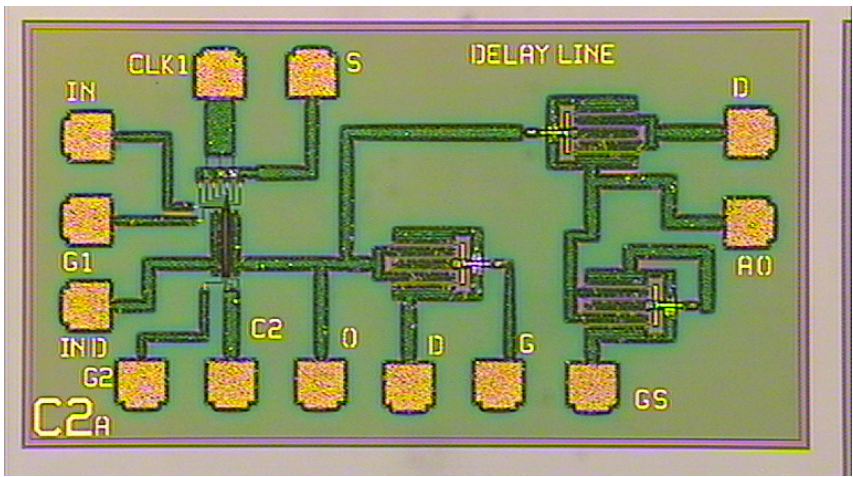

Fig.13. The fabricated 13-gate CCD delay line based on the $2^{\text {nd }}$ device structure as shown in Fig. 12.

The reset transistor in the charge sensing circuitry acts as a charge extraction device while the source follower which has a unity gain, accurately coverts the signal charge arriving at the output of the CCD to a voltage waveform. The charge will be attracted to the output ohmic contact due to the positive 'floating' voltage level which exists at this ohmic gate contact and once this signal charge has been sensed, it will then be extracted out to make way for new charge packets.

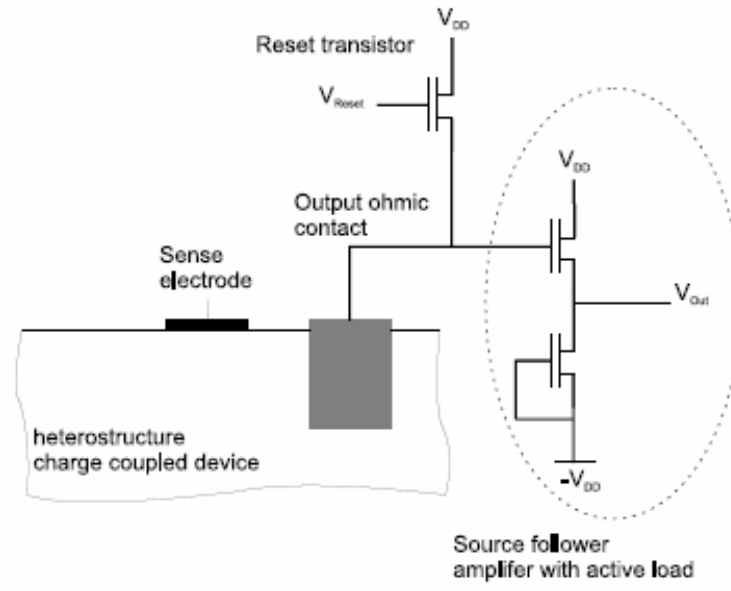

Fig.14. The charge sensing and extraction circuitry which is connected to the output diffusion of the CCD.

\section{DC CHARACTERIZATION}

The CCD described in the previous section is effectively a pHEMT with the drain and source ohmic contacts separated by three groups of independently accessed Schottky gates. It should also be noted that the drain-source contacts are separated by 12 individual gates totaling $28 \mu \mathrm{m}$ in length from the drain to source contact. When the CCD delay line is operated as a pHEMT, this allows for the verification of the charge transfer process within the device as well as providing some insight into the device's operating characteristics such as its drain-gate and drain-source breakdown values.

A programmable power supply (HP6626A) was used to measure the DC characteristics of the CCD. Fig. 15 shows the measured current-voltage characteristics of the CCD 
it is operated in 'transistor mode', where all the gates are bonded together and driven from 0 to -1 volt. Successive decreases in the maximum obtainable drain-source current were observed when the gates were driven with increasingly negative voltages.

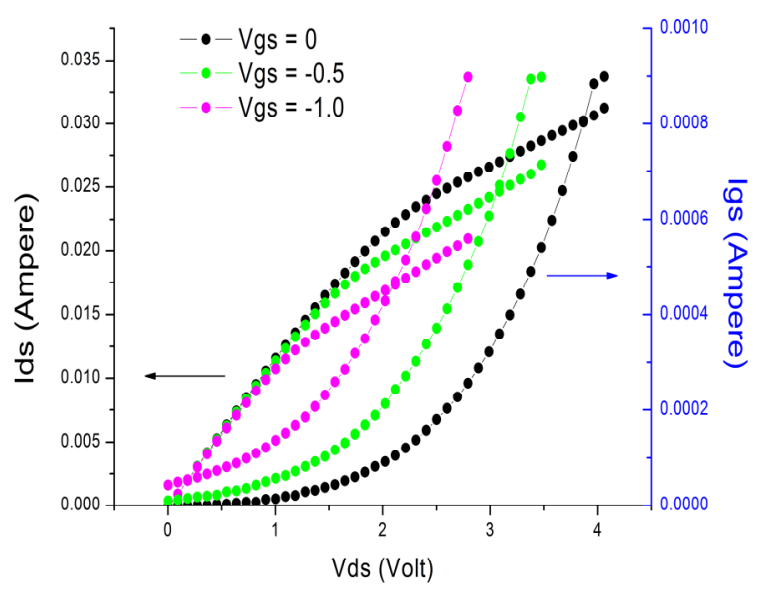

Fig.15. The measured current-voltage curves for the $1^{\text {st }}$ device structure CCD delay line when it is operated as a pHEMT.

\section{CONCLUSIONS}

A detailed study and simulation of GaAs-based CCD structures has been presented. The relationship between the 'potential wells' and carrier concentration in the channel which were obtained using the quasi-2D model, have demonstrated that 2DEG-CCD structures which were based around commercial pHEMT structures could potentially be used as delay line devices if the appropriate device structure is chosen. These 2DEG-CCD delay lines when combined with split-electrode gate structures [13] have the potential to be used to design tunable MMIC charge transversal filters as described in [5].

\section{ACKNOWLEDGMENT}

The authors would like to acknowledge useful discussions with Dr. David Denis. Special thanks also go to Filtronic Plc. for sponsoring this research.

\section{REFERENCES}

[1] R. A. LaRue, R. E. Colbeth, G. A. Davis, C. Yuen, C. Webb, C. Shih, and R. E. Weiss, "Performance of modulation doped charge coupled devices (MD-CCD's) in the microwave and millimeter wave bands," IEEE Transactions on Electron Devices, vol. 41, pp. 10-18, 1994.

[2] E. R. Fossum, J. I. Song, and D. V. Rossi, "Two dimensional electron GAS charge coupled devices (2DEG-CCDs)," IEEE Transactions on Electron Devices, vol. 38, pp. 1182-1192, 1991.

[3] Y. Z. Liu, I. Deyhimy, R. J. Anderson, J. S. Harris Jr, and L. R. Tomasetta, "AlGaAs/GaAs heterojunction Schottky barrier gate CCD," Technical Digest, IEDM, pp. 622-624, 1979.

[4] H. T. Tan, I. C. Hunter, C. M. Snowden, and R. Ranson, "Design and fabrication of modulation doped charge coupled devices for transversal filter applications," presented at The 10th High Frequency Postgraduate Student Colloquium, Leeds, 2005.

[5] H. T. Tan, I. C. Hunter, C. M. Snowden, and R. Ranson, "Design and fabrication of short gate-length heterostructure charge coupled devices for transversal filter applications," presented at European Gallium Arsenide and other Compound Semiconductors Application Symposium, Paris, 2005.

C. K. Kim and M. Lenzlinger, "Charge transfer in charge coupled devices," Applied Physics Letters, vol. 17, pp. 111-115, 1970.

[7] J. D. E. Beynon and D. R. Lamb, "CCD circuit techniques for analogue signal processing," in Charge coupled devices and their applications, vol. 1. London: McGraw-Hill Book Company (UK) Limited, 1980, pp. 182-216.

[8] C. M. Snowden, "Characterization and Modeling of Microwave and Millimetre-wave large signal device circuit interaction based on electro-thermal physical models," presented at IEEE Int. Microwave Symposium, Denver, USA, 1997.

[9] R. Drury and C. M. Snowden, "A quasi two dimensional HEMT model for microwave CAD applications," IEEE Transactions on Electron Devices, pp. 1026-1032, 1995.

[10] C. Morton, J. Atherton, C. M. Snowden, R. Pollard, and M. J. Howes, "A large-signal physical HEMT model," IEEE MTT-S International Microwave Symposium, pp. 1759-1762, 1996.

[11] C. Morton, "The epitaxial layer design of HEMTS." York: University of York, 1994.

[12] R. Drury, "The physical modelling of heterojunction field effect transistors," in Electrical and Electronics Engineering. Leeds: University of Leeds, 1993.

[13] R. D. Baertsch, W. E. Engeler, H. S. Goldberg, C. M. Puckette, and J. J. Tiemann, "The design and operation of practical charge-transfer transversal filters," IEEE Journal of Solid-State Circuits, vol. SC-11, pp. 65-74, 1976. 